\title{
Measuring Resource Inequality: The Gini Coefficient
}

\author{
Michael T. Catalano \\ Dakota Wesleyan University, micatala@dwu.edu \\ Tanya L. Leise \\ Amherst College, tleise@amherst.edu \\ Thomas J. Pfaff \\ Ithaca College, tpfaff@ithaca.edu
}

Follow this and additional works at: https://digitalcommons.usf.edu/numeracy

Part of the Mathematics Commons, and the Science and Mathematics Education Commons

\section{Recommended Citation}

Catalano, Michael T., Tanya L. Leise, and Thomas J. Pfaff. "Measuring Resource Inequality: The Gini Coefficient." Numeracy 2, Iss. 2 (2009): Article 4. DOI: http://dx.doi.org/10.5038/1936-4660.2.2.4 


\title{
Measuring Resource Inequality: The Gini Coefficient
}

\begin{abstract}
This paper stems from work done by the authors at the Mathematics for Social Justice Workshop held in June of 2007 at Middlebury College. We provide a description of the Gini coefficient and some discussion of how it can be used to promote quantitative literacy skills in mathematics courses. The Gini Coefficient was introduced in 1921 by Italian statistician Corrado Gini as a measure of inequality. It is defined as twice the area between two curves. One, the Lorenz curve for a given population with respect to a given resource, represents the cumulative percentage of the resource as a function of the cumulative percentage of the population that shares that percentage of the resource. The second curve is the line $y=$ $x$ which is the Lorenz curve for a population which shares the resource equally. The Gini coefficient can be interpreted as the percentage of inequality represented in the population with respect to the given resource. We propose that the Gini coefficient can be used to enhance students' understanding of calculus concepts and provide practice for students in using both calculus and quantitative literacy skills. Our examples are based mainly on distribution of energy resources using publicly available data from the Energy Information Agency of the United States Government. For energy resources within the United States, we find that by household, the Gini coefficient is 0.346 , while using the 51 data points represented by the states and Washington D.C., the Gini coefficient is 0.158 . When we consider the countries of the world as a population of 210 , the Gini coefficient is 0.670 . We close with ideas for questions which can be posed to students and discussion of the experiences two other mathematics instructors have had incorporating the Gini coefficient into pre-calculus-level mathematics classes.
\end{abstract}

\section{Keywords}

Mathematics, Quantitative Literacy, Inequality, Gini Coefficient, Calculus, Social Justice, Inequity, Lorenz Curves, Energy, Energy Resources, Resource Allocation

\section{Creative Commons License}

$$
\text { (c) (1) (5) }
$$

This work is licensed under a Creative Commons Attribution-Noncommercial 4.0 License 


\section{Introduction}

Today, there is a huge disparity between the technology, education, health care and agricultural methods that are available in the developed and developing world. The principal challenge we face is to close that gap ... The countries, businesses and individuals that are on the right side of the divide have to think hard about what kind of world they want us all to live in 20 years from now. Narrowing the gap benefits everyone, and we have the means to do it. If we don't, we will have missed an amazing opportunity.

- Bill Gates (Hofheinz 2000)

Inequality, or disparity using Gates' terminology, is a recurring topic within political and social discourse. Whether it is technology, education, health care, or (most importantly in most peoples' minds) money, we know that some have more and some have less, the latter sometimes arguably having not enough. How unfair the inequality is with respect to any particular resource is a matter of debate. However, before engaging in the fairness debate, there is the matter of how one actually measures inequality. The Gini coefficient, introduced by Italian statistician Corrado Gini in 1921 (Gini 1921), has been used in a wide variety of resource allocation contexts to measure inequality including income, wealth, credit availability, health care, and energy (Berndt et al. 2003).

\section{The Gini Coefficient and Quantitative Literacy}

In this article, we describe the concept of the Gini coefficient, demonstrate how to calculate it, and provide examples involving energy consumption inequity. We discuss how this material can be used to enhance calculus courses in particular, but also include some ideas for the pre-calculus level, for example, algebra and modeling courses. Our goals in undertaking this project were to develop and disseminate a set of examples that could be used to promote awareness of social justice issues among students taking mathematics classes, as well as provide opportunities for students to learn and practice quantitative literacy skills.

Given this latter goal, it might be appropriate for us to say how we conceive of quantitative literacy and what role it might play within the calculus curriculum. Quantitative literacy is not a well-defined concept, 
nor is there consensus within the mathematical community on what constitutes quantitative literacy. However, we consider the following skills to be part of what it means to be quantitatively literate:

1. The ability to understand quantitative information within a variety of real-world contexts, including the relevance and meaning of the given quantitative information in that context.

2. The ability to use mathematical and statistical methods to increase understanding, solve problems, and reach conclusions within a variety of real-world contexts. This would include being able to use techniques from different areas of mathematics within a single context.

3. The ability to critique the reasonableness of statements that include quantitative information or have quantitative information as support.

4. The ability to use and develop appropriate representations of quantitative information and use these within the context of written and oral communications.

5. The ability to understand mathematical and quantitative content from a conceptual standpoint, not simply as a set of algorithms or procedures.

Notice that these items say very little about what particular mathematical skills should be considered a part of quantitative literacy, other than specific mention of statistics. We believe students, at whatever level, should be encouraged and able to use the skills they have to understand and learn about the world around them. The 20th century artist Robert Henri described this as the student being "master from the beginning; that is, he must be master of such as he has." ${ }^{1}$ One implication of this perspective is that quantitative literacy need not, in fact should not, be confined to a narrow set of quantitative literacy courses at the pre-calculus level. It should pervade not only the college curriculum but also, and perhaps more so, the K-12 curriculum. Many of our college students are not going to take calculus and so it is important for students who are only going to take a single pre-calculus level class to be exposed to quantitative literacy. However, this does not

\footnotetext{
${ }^{1}$ p. 12, The Art Spirit, by Robert Henri
} 
mean we should ignore the quantitative literacy needs of our calculus students.

Please also note that in our view quantitative literacy is not primarily about the ability to carry out particular calculations, whether in arithmetic or algebra or calculus. While learning fundamental mathematical skills is a necessary prerequisite for gaining quantitative literacy, it should be the means to an end, rather than the principal goal of mathematical education. The ability to analyze quantitative information in a real-world context is the skill that will enable a person to navigate more successfully through life in our information society. The knowledge of how to carry out certain calculations is not sufficient in itself. For example, a calculus student who is quite good at taking derivatives, but is unable to apply the concept of rate of change beyond textbook exercises, is missing key quantitative literacy skills. In whatever mathematics course we teach, at whatever level of the curriculum, if we hope to improve our students' quantitative literacy, we must help them move past focusing on the calculations themselves to thinking about how their new quantitative tools can aid them in understanding our complicated world.

In the next sections we describe how to cover the Gini coefficient and related material in a calculus course to address items 4 and 5 above, and to a lesser extent, items 1 and 2. As we will see when we get into the details below, the Gini coefficient is a way to represent or measure inequality as the area between two curves. Understanding the Gini coefficient requires students to have a conceptual understanding of area as well as cumulative percentiles. Students at the pre-calculus level can certainly appreciate the application of the concept of area to represent inequality, even if they use non-calculus methods to calculate or estimate the area. For calculus students, this material provides students with the opportunity to study the meaning of the derivative within a new conceptual context, and reinforces the idea that integration is a tool that allows us to measure area between curves precisely. The manner of calculation is not the central focus here; it is using the students' current set of mathematical tools to analyze a quantitative issue arising in the real world.

We would also like to mention the aspect of social justice in our motivation regarding this project. Incorporating social justice into the mathematics curriculum almost necessitates the use of quantitative literacy on the part of the students. It is one of many possible contexts in which quantitative information arises. The use of social justice issues 
can increase the emotional investment students have in their learning, depending on the particular issue. It allows students to make connections between their mathematical learning, what they learn in other courses and from news and other media, and their pre-existing knowledge and attitudes. We believe these types of connections can greatly enhance the value and depth of the learning students experience in our classes.

\section{Introduction to Lorenz Curves and the Gini Coefficient}

We begin discussion of the details of the Gini coefficient with an illustrative example involving discrete data; continuous models will be introduced later. Suppose we have 100 tokens (each representing a unit of energy, income, food, water, or some other resource) and we divide them up among 10 people, giving the first 1, the second 3, etc., so that the $k$ th person is assigned tokens according to the distribution function $u(k)=2 k-1$. Note that in this distribution we are ranking the people in ascending order according to number of tokens assigned. Also note that there is considerable inequality here: one individual receives only a single token, whereas another individual is showered with 19 tokens.

Given this distribution function, we can define the associated Lorenz curve, $L(x)$, as the graph of the cumulative proportion function. ${ }^{2}$ In other words, $L(x)$ is the proportion of tokens held by the poorest $100 x \%$ of the population. In our example, $L(0.1)=1 / 100, L(0.2)=4 / 100$, and in general, $L(x)=x^{2}$ for all $x=0,0.1,0.2, \cdots 1$. So, for example, $L(0.5)=0.25$ which indicates that the bottom $50 \%$ of the population has $25 \%$ of all the tokens. The top $10 \%$ of the population receives $1-L(0.9)=19 / 100$ or $19 \%$ of the tokens.

Now, if we wanted to distribute our 100 tokens as equitably as possible, we would assign each of the 10 people the same amount, namely ten tokens. The distribution function would be $u(k)=10$. The associated Lorenz curve is formed by connecting the set of discrete data points $(x, L(x))$ via a piecewise linear function or best-fit curve. In the case of a uniform distribution function, we have $L(x)=x$. Thus,

\footnotetext{
${ }^{2}$ The Lorenz curve is named after Max Otto Lorenz, an American economist who published the idea in 1905 to describe income inequality (Lorenz 1905). Max Lorenz should not be confused with Edward Lorenz who founded chaos theory, Konrad Lorenz who studied animal behavior, or the physicist Hendrik Lorentz.
} 
the function $L(x)=x$ represents perfect equality. On the other hand, if we wanted to create more inequality than represented by our first distribution $u(k)=2 k-1$, we might instead use the distribution $u(k)=2^{k-1}$, so that the first person still receives one token, the second two, and the 10 th receives $2^{9}=512$. This will require a total of $2^{10}-1=1023$ tokens, but that is okay since we are going to consider the proportion of tokens anyway. For $x=0.1,0.2, \cdots 1$, we have that $L(x)=\frac{2^{10 x}-1}{1023}$. In this case, $L(0.1)=1 / 1023, L(0.5)=31 / 1023 \approx .03$, and $L(0.9)=511 / 1023 \approx 0.5$. Thus, the bottom $50 \%$ of the population receives only $3 \%$ of the tokens, the bottom $90 \%$ receives about half, and the top $10 \%$ also receives about half.

Graphs for all three of these Lorenz curves appear in Figure 1. Observe that increasing the disparity among tokens received by different individuals pushed the Lorenz curve further away from the "equality curve" $y=x$. One reasonable way to measure the relative disparity of token distribution is the area between $y=x$ and any other given Lorenz curve. The ratio of this area to the total area of the triangle under $y=x$ is how the Gini coefficient is defined. Note that in defining the Gini coefficient in this way we are implicitly expanding the domain of our Lorenz functions $L(x)$ to the interval $[0,1]$. Under this definition, the Gini coefficient will be at least 0 and at most 1 . The latter situation arises if one person within the population gets all the tokens, and everybody else gets none.

Now imagine distributing tokens among a very large population. In the extreme case of an infinitely divisible population, so any proportion between 0 and 1 can be made, we can assume the Lorenz curve is continuous and apply calculus to calculate the Gini coefficient. Recall that the Lorenz curve shows the cumulative proportion of tokens owned by a proportion of the population. In calculus notation, the Gini coefficient equals $\int_{0}^{1}(x-L(x)) d x$ divided by $\frac{1}{2}$. This simplifies to

$$
\text { Gini coefficient }=1-2 \int_{0}^{1} L(x) d x .
$$

Clearly, the uniform distribution with $L(x)=x$ results in a Gini coefficient of 0 , representing perfect equity (zero inequity). For the other two examples, we can use any of the standard calculus techniques, either numerical or symbolic, for determining the area between two curves. In cases where $L(x)$ itself is given only numerically, we could find an appropriate best-fit curve for each example and integrate that function. Examples utilizing these various methods are given later in this paper. 
Three Lorenz Curves

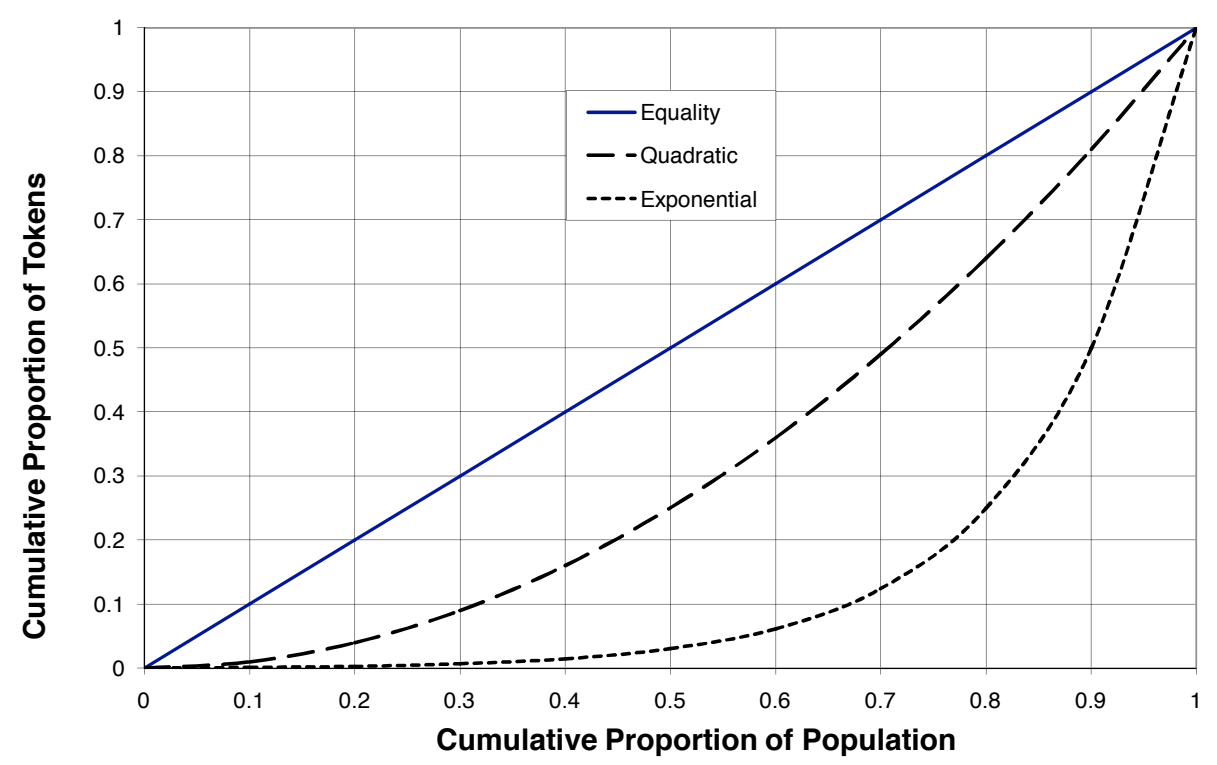

Figure 1: An example of three possible Lorenz curves, $L(x)=x$ represents equality, while $L(x)=x^{2}$ and $L(x)=\left(2^{10 x}-1\right) / 1023$ represent two different inequality scenarios.

There are also more specialized methods, such as the numerical rule based on quintiles developed by Leon Gerber (Gerber 2007).

For the examples shown in Figure 1, the Gini coefficients can be calculated using calculus by treating the Lorenz curve $y=L(x)$ as a continuous function. For $L(x)=x^{2}$, we get a Gini coefficient of

$$
1-2 \int_{0}^{1} x^{2} d x=\frac{1}{3}
$$

For the second example, the Gini coefficient is

$$
1-2 \int_{0}^{1} \frac{2^{10 x}-1}{1023} d x=1-2\left(\frac{2^{10}-1}{10230 \ln 2}-\frac{1}{1023}\right) \approx 0.7134 .
$$

As expected, the second example has a much larger coefficient, reflecting the greater intuitive inequality. We could say the first distribution is fairer than the second. In practical terms, however, would we say that both of these distributions are unfair, or is the first fair and not 
the second, or are they perhaps both to be considered reasonably fair or at least not problematical?

With respect to income, the United Nations suggests that a national Gini coefficient larger than 0.40 is considered high (People's Daily Online 2006). Although they vary over time, Gini coefficients for income within countries tend to range from a little under 0.20 to around 0.60 , with 0.40 being somewhat above the median. On the other hand, if one calculates the Gini coefficient for the entire world considered as a single population, the current value would be in the range of 0.65 (Palma 2006).

The Gini coefficient is highly dependent on how the population is selected. For example, Tang et al. (Chotikapanich et al. 2006) note that the Gini coefficient for urban residents of China and rural residents of China were both roughly 0.33 in 2003. However, the index for the country as a whole was over 0.40. This was because the disparity between the groups was larger than the disparity within the two separate groups. Gini indices are typically affected by quite a number of hidden variables, including household size, age distribution within a population, and discrepancies between countries on how income data are collected. In addition, as an economy undergoes certain types of changes, inequality can increase, even though these changes might be beneficial to the society as a whole and possibly even for the subgroups that are "falling behind." For example, if a country experiences an upsurge in high paying jobs based on the new "knowledge economy," inequality will increase even though potentially no one in the country is actually worse off (Kwok-chuen 2007).

The Gini coefficient can be used to measure inequality in many other contexts besides income, including wealth, education, energy consumption (Jacobson et al. 2007), airport concentration, plant sizes and weights, or any other measurable quantity distributed across a population. The Gini coefficient is sometimes interpreted as the percent of inequality within a given population, and in this context it is called the Gini index. For further discussion of the merits of the Gini coefficient compared to other measures of inequality, see University of Texas Inequality Project (2007) and Palma (2006). Lastly, we note that the Thiel index (see University of Texas Inequality Project 2007) is also a frequently used, if technically involved, measure of inequality that has some advantages over the Gini coefficient. 


\section{Calculus and Lorenz Curves}

The use of best-fit curves to replace the discrete $L(x)$ function allows us to work in the calculus context, as we did previously, so we proceed with continuous functions for $u$ and $L$, rather than with discrete data. Suppose we have a continuous distribution function $u(x)$ on $[0,1]$ describing, for example, energy consumption of a population. In this context, $u(0.2)$ indicates the 20th-percentile energy consumption level: the energy consumption of $20 \%$ of the population is less than or equal to $u(0.2)$, while that of $80 \%$ is at least $u(0.2)$. The function $u(x)$ is assumed to be nonnegative and nondecreasing, that is, $u(x) \geq 0$ and $u^{\prime}(x) \geq 0$, since we order the population from poorest to richest in resources, as was done in the previous examples. The normalized cumulative distribution function is

$$
L(x)=\frac{1}{u_{m}} \int_{0}^{x} u(s) d s,
$$

where $u_{m}=\int_{0}^{1} u(s) d s$ is the mean value of $u(x)$ on $[0,1]$. The Lorenz curve is the graph of $L(x)$ on the interval [0,1]. Although both the distribution functions $u(x)$ and the Lorenz function $L(x)$ need not be everywhere differentiable - for instance it is possible for $L(x)$ to be piecewise continuous - in practice we will usually be approximating discrete data with functions that are differentiable. Statements regarding the derivatives of $u(x)$ and $L(x)$ should be taken in this context.

Basic properties of the Lorenz curve:

1. $L(0)=0$ and $L(1)=1$ : This is immediate from the definition.

2. $L(x)$ is nondecreasing: $L^{\prime}(x)=u(x) / u_{m} \geq 0$.

3. $L(x)$ is concave up: $L^{\prime \prime}(x)=u^{\prime}(x) / u_{m} \geq 0$.

4. The slope of the Lorenz curve will equal 1 either at a unique point or on a single closed interval: Recall that $u$ is nondecreasing, so $0 \leq u(0) \leq u_{m} \leq u(1)$. This implies that $0 \leq L^{\prime}(0)=u(0) / u_{m} \leq$ 1 and $L^{\prime}(1)=u(1) / u_{m} \geq 1$. Since $L^{\prime \prime}(x) \geq 0$, there must be an interval $[a, b] \subset[0,1]$ (where $a=b$ is possible) on which $L^{\prime}(x)=1$, while $0 \leq L^{\prime}(x)<1$ on $[0, a)$ and $L^{\prime}(x)>1$ on $(b, 1]$.

5. This point or interval where $L^{\prime}(x)=1$ partitions the population in a natural way: 
(a) If $L^{\prime}(x)<1$, then $u(x)=u_{m} L^{\prime}(x)<u_{m}$ (below average resource consumers).

(b) If $L^{\prime}(x)=1$, then $u(x)=u_{m} L^{\prime}(x)=u_{m}$ (average resource consumers).

(c) If $L^{\prime}(x)>1$, then $u(x)=u_{m} L^{\prime}(x)>u_{m}$ (above average resource consumers).

We should note that this gives a straightforward way to derive Lorenz curves, but the more typical approach would be to use density functions, as is presented by Leslie (2007). However, the latter approach is much more involved and potentially confusing. In fact, in the examples we give below we will start with cumulative data, and use curve fitting in Excel to find $L(x)$ as a practical way to obtain Lorenz curves.

\section{Three Energy Gini Examples}

In this section we provide three examples of calculating the Gini coefficient for energy consumption. ${ }^{3}$ In each of our examples we start with cumulative data and use Excel to find a fitted curve to approximate the Lorenz curve. We provide one data set here so that readers can consider the example on their own using the technology of their choice.

The first example is per capita 2001 U.S. electricity consumption (Energy Information Agency 2004). The data are compiled from a sample of 4822 households. We reduce the data to the deciles in Table 1 before curve fitting. The cubic curve we get from Excel (enforcing the condition $L(0)=0$ via the intercept option) for these data is $L_{\text {USpop }}(x)=0.6408 x^{3}+0.0326 x^{2}+0.3116 x\left(r^{2}=0.999\right)$. The next example uses data on 2004 electricity consumption per person by state in the U.S. (Energy Information Agency 2007a). ${ }^{4}$ The 51 data points generate a Lorenz curve of $L_{\text {state }}(x)=0.4678 x^{2}+0.5305 x$ $\left(r^{2}=1\right)$. The last example again uses data on 2004 Per Capita Total Primary Energy Consumption by country (Energy Information Agency 2007b) . Here we use 210 data points to find the fitted curve of $L_{\text {world }}(x)=7.8995 x^{5}-16.299 x^{4}+12.358 x^{3}-3.4276 x^{2}+0.3221 x$ $\left(r^{2}=0.994\right)$. In these examples the condition $L(0)=0$ is satisfied but

\footnotetext{
${ }^{3}$ The data are available at www.ithaca.edu/tpfaff/sustainability.htm in an Excel format.

${ }^{4}$ The EIA website has recently replaced the 2004 data with updated 2006 data.
} 


\section{Table 1: Deciles of U.S. Household Electricity Consumption}

\begin{tabular}{|c|c|}
\hline Proportion of Population & $\begin{array}{c}\text { Cumulative Proportion of } \\
\text { Electricity Consumption }\end{array}$ \\
\hline 0.0 & 0.000 \\
\hline 0.1 & 0.023 \\
\hline 0.2 & 0.060 \\
\hline 0.3 & 0.110 \\
\hline 0.4 & 0.175 \\
\hline 0.5 & 0.254 \\
\hline 0.6 & 0.345 \\
\hline 0.7 & 0.459 \\
\hline 0.8 & 0.588 \\
\hline 0.9 & 0.754 \\
\hline 1.0 & 1.000 \\
\hline
\end{tabular}

the conditions that $L(1)=1$ and that $L(x)$ be increasing on $[0,1]$ are not always met. By using mathematical software like Mathematica or Maple, one can enforce all of these conditions. However, the effect on the Gini coefficients (listed in Table 2) is quite small, and Excel has the advantage of being more broadly accessible.

The Gini coefficients for these three curves, calculated using (1), are in Table 2. One should certainly ask if the relative values of these coefficients is expected. In other words, should it be the case the

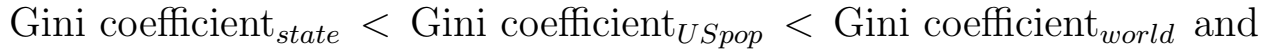
if so why?

\section{Table 2: Three Gini Coefficients}

\begin{tabular}{|c|c|}
\hline Lorenz Curve & Gini coefficient \\
\hline$L_{\text {USpop }}(x)$ & 0.346 \\
\hline$L_{\text {state }}(x)$ & 0.158 \\
\hline$L_{\text {world }}(x)$ & 0.670 \\
\hline
\end{tabular}

We can also use a numerical estimate instead of curve fitting to calculate the Gini coefficient. We will use the trapezoid rule and the data from Table 1. From Table 3 we estimate that the area under the 
curve is $0.32681 \approx 0.327$. However, recall that this is not the Gini coefficient but an estimate of the area under the cumulative proportion of electricity consumption data. The Gini coefficient is given by $1-$ $2(0.32681) \approx 0.346$, which happens to agree quite well with the curvefitting estimate.

Table 3: Trapezoid rule calculation of the Gini coefficient using the data in Table 1.

\begin{tabular}{|c|c|c|c|}
\hline $\begin{array}{c}\text { Proportion of } \\
\text { Population }\end{array}$ & $\begin{array}{c}\text { Cum. Prop. of } \\
\text { Elect. Consum. }\end{array}$ & $\begin{array}{c}\text { Average of } \\
\text { Consec. } y \text { 's }\end{array}$ & $\begin{array}{c}\text { Area of } \\
\text { Trapezoids }\end{array}$ \\
\hline 0.0 & 0.000 & 0.01155 & 0.001163980 \\
\hline 0.1 & 0.023 & 0.04155 & 0.004155 \\
\hline 0.2 & 0.060 & 0.0850 & 0.00850 \\
\hline 0.3 & 0.110 & 0.1425 & 0.01425 \\
\hline 0.4 & 0.175 & 0.2145 & 0.02145 \\
\hline 0.5 & 0.254 & 0.2995 & 0.02995 \\
\hline 0.6 & 0.345 & 0.4020 & 0.04020 \\
\hline 0.7 & 0.459 & 0.5235 & 0.05235 \\
\hline 0.8 & 0.588 & 0.671 & 0.0671 \\
\hline 0.9 & 0.754 & 0.877 & 0.0877 \\
\hline 1.0 & 1.000 & \multicolumn{2}{|c|}{ Sum $=0.32681$} \\
\hline
\end{tabular}

Instructors can use these data and similar data in a classroom in a number of ways. Students can be given the Lorenz curves directly or given the data to calculate the Gini coefficient by a numerical method or through curve fitting and integration. Either way, there is a number of questions and activities that can be pursued. For example students can be asked to find a best-fit curve for given data and then determine where the Lorenz curve has a slope of 1, along with interpreting what this point means. For instance, using $L_{U \text { Spop }}(x)$ we solve $L_{U \text { Spop }}^{\prime}(x)=1$ to get $x=-0.518$ and $x=0.585$. In this example the $x=-0.518$ has no interpretation. On the other hand we can say that, according to this model, the bottom $58 \%$ of energy consumers are using less than their allotted share while the top $41 \%$ are using more. 


\section{Suggestions for Further Exploration}

A few other thought-provoking exercises are the following. If two Lorenz curves have the same Gini coefficient, must these Lorenz curves be the same? If so, explain why. If not, sketch two different Lorenz curves that yield the same Gini coefficient. Can two Lorenz curves intersect and still give the same Gini coefficient and is there a limit on the number of isolated intersections possible? Can you have two Lorenz curves $f(x)$ and $g(x)$ with $f^{\prime}(x)>g^{\prime}(x)$ for all $x$ in $(0,1)$ ?

Other suggestions for further exploration include:

1. Having follow-up discussion of implications of Gini coefficients. What types of individuals or sectors of the economy might be using more energy than necessary? What sectors of the economy might be targeted for energy conservation measures?

2. Finding other data to analyze, for example, petroleum consumption, residential use versus industrial use, comparison using states in US as the individuals. The Energy Information Administration website has lots of pertinent data to explore.

3. Finding other areas where Gini coefficient might be applicable (income disparity, basketball salaries, portfolio analysis, etc).

4. Asking what might be sensible thresholds for reasonably equitable energy distribution and for extremely skewed energy consumption.

5. Reflecting on ramifications of inequalities in energy consumption. What might be advantages and disadvantages of inequality?

Other exercises and activities can be found in Cheung et al. (2005), Leslie (2007), Teague (2004), University of Texas Inequality Project (2007), and Zorn (2005).

\section{Pre-calculus Possibilities}

While the discussion above has focused on calculus, one can certainly bring the Gini coefficient into pre-calculus-level courses. Co-author Catalano has incorporated the Gini coefficient into a college algebra text that is under development. ${ }^{5}$ The major adaptation is students will

\footnotetext{
${ }^{5}$ This work has been supported by an NSF CCLI-EMD grant, DUE-0442979.
} 
need to use an alternative method to estimate the area between the curves. There are several ways this can be done:

1) In many cases, inequality data of various sorts are available by quintiles or deciles, as in our energy examples. Students could use trapezoids (essentially the trapezoid rule from calculus) to estimate the area between curves based on data in either of these formats.

2) A more intuitive, if less accurate, method would be to have students create graphs on Excel including appropriately scaled grid lines. Students could then estimate the area by "counting grid rectangles."

3) A second intuitive method would be to have students create a triangle that has roughly the same area as the given area based on the Lorenz curve. The base of the triangle would be along the $x$-axis from zero to a given cumulative population percentile $p$ and the second two sides would run from the endpoints of the base to the point $(1,1)$. The triangle would thus have height one and the length of the base would equal $p$. The estimated Gini coefficient would then be twice the area of this triangle or $2(1 / 2) b h=p$. For example, in considering a curve like the exponential curve in Figure 1 above, students would reasonably run the base of the triangle from 0 to 0.7, giving an estimated Gini coefficient of 0.7. Recall the coefficient calculated via calculus is 0.7134 .

Catalano has had students utilize both methods 2 and 3 in his college algebra courses. In using method 3, students are asked to actually draw in a triangle on the graph of the Lorenz curve. Most students are able to understand the idea of equivalent but differently shaped areas and use the area formula for the triangle to make reasonable estimates. In general, students who make errors tend to overestimate the area, sometimes drawing triangles which include the entire area between the Lorenz curve and the equality line.

Other activities Catalano has had students work on have included:

1) Create a Lorenz curve where the bottom half of the population receives none of the resource and the top half of the population shares the resource equally. 
2) Create a Lorenz curve where one person uses half of the resource and the remainder of the population shares half of the resource equally.

3) Create a Lorenz curve where the bottom half of the population shares $p \%$ of the resource equally and the top half of the population shares the remainder of the resource equally.

4) Estimate the Gini coefficient in each of the three scenarios above.

Since these examples all involve piecewise linear functions, the area calculations can all be done via triangles and elementary geometry.

\section{Concluding Remarks}

Catalano has found that working with the Gini coefficient certainly presents students with a use of area that is novel for them. Most students can appreciate the idea of inequality and the sharing of percentages of a resource. Students are often astonished at how small of a percentage the poorest fifth of a population shares, and how large a percentage the top fifth obtains. When presented with other examples of Gini coefficient data or Lorenz curves, students appreciate understanding how these data are determined. Especially in a course where various types of indices (Dow Jones Industrial Average, Consumer Price Indices, etc.) are discussed, exposing students to how such indices are developed provides a deeper quantitative literacy education. Certainly having students understand the advantages and limitations of a given numerical measure is an important quantitative literacy goal.

Although unrelated to our present work on energy resources, we would also like to mention the experiences of two colleagues. The first is Charlie Hadlock, who has used the Gini Coefficient in a mathematical modeling course at Bentley College. ${ }^{6}$ In addition to having students collect information and calculate Gini coefficients for collections of countries with respect to wealth and income, Hadlock also engaged students in discussion of what an acceptable threshold of inequality might be, both with respect to ethical considerations and from the standpoint of avoiding social unrest. Students considered how various factors (inheritance rules, level of economic trade, etc.) affected

\footnotetext{
${ }^{6}$ Dr. Hadlock is a faculty member at Bentley College in Waltham, Massachusetts, and provided us this information in a personal communication.
} 
inequality within artificial societies. Students either wrote their own programs to do the calculations or used a previously developed Netlogo model. Hadlock's students found that their Gini calculations varied depending on what assumptions were made about the behavior of the Lorenz curves between the small number of data points typically available, eventually convincing the team of Netlogo developers to modify their assumptions for their Gini coefficient model. Hadlock indicated that his students appreciated the "cleverness" of the area approach inherent in the Gini coefficient and that they did some very worthwhile thinking and learning in their constructions and calculations.

Secondly, Andrew Miller includes discussion of the Gini coefficient as a measure of income inequality in a general education course at Belmont University ("mathematics for liberal arts") as part of a unit on money and economics. ${ }^{7}$ When he asked students to comment on this material, the great majority enjoyed the unit, and its inclusion in the course appeared to increase the number of students who believed the course would be beneficial to their future studies or to their life after college. Miller says that he deliberately presents the Gini coefficient material in a neutral manner, in order to let the students interpret this measure of income inequality for themselves. This raises an important point: coverage of mathematics relating to social issues by mathematicians is best handled in a neutral manner, presenting the data and the quantitative analysis in an objective fashion. Reflection through discussion in class can then be mediated by the instructor, rather than dictated by the instructor.

While the authors have not as yet tested our energy examples in a classroom setting, we believe previous experiences show that utilizing the Gini coefficient can serve to help students appreciate the applicability of calculus techniques, as well as provide practice for students in using quantitative literacy skills and calculating areas between curves in a context-rich setting using both symbolic and numerical techniques. AMATYC (1995) recommends that students "learn mathematics through modeling real-world situations, ... develop the view that mathematics is a growing discipline, interrelated with human culture, ... [and] use appropriate technology to enhance their mathematical thinking and understanding and to solve mathematical problems and judge the reasonableness of their results." Although AMATYC

\footnotetext{
${ }^{7}$ Information provided by Dr. Miller, a faculty member at Belmont University in Nashville, TN, via a personal communication.
} 
addressed pre-calculus classes, we believe quantitative literacy goals are also appropriate for calculus classes, especially where these courses fulfill a general education mission.

In addition, through discussion and reflection combined with the mathematical exercises, the Gini coefficient can be effective in increasing students' awareness of the inequity of energy consumption and more generally the distribution of wealth and resources (Staples 2005), as well as in reinforcing the importance of mathematical metrics regarding important social issues.

\section{Acknowledgments}

The authors would like to acknowledge the work of Priscilla Bremser, Chawne Kimber, and Karen Levin in hosting the Second Workshop on the Mathematics of Social Justice at Middlebury College in June of 2007. Work on this project began at that conference, and also provided the venue for the authors to become acquainted. The Workshop was made possible by a grant from the Learn and Serve America Corporation, through the Pennsylvania/New York Campus Compact Consortium. Matching funds were provided by Lafayette College, Middlebury College, Moravian College, West Chester University, and the University of East Stroudsburg. Also, thanks to Daniel L. King for helpful discussions.

\section{References}

AMATYC (American Mathematical Association of Two-Year Colleges). 1995. Crossroads in Mathematics: Standards for Introductory College Mathematics Before Calculus. Memphis, TN: AMATYC.

Berndt, D. J., J. W. Fisher, R. V. Rajendrababu, and J. Studnick. 2003. Measuring Health Care and Inequities Using the Gini Index. 36th Annual Hawaii International Conference on System Sciences: p. 159.3.

Cheung, C. S. , C. C. Kwan, and P. C. Miu. 2005. Mean-Gini Portfolio Analysis: A Pedagogic Illustration, Spreadsheets in Education. 2(2): 194-207.

Chotikapanich, D., D.S. Prasada Rao, and K. K. Tang. 2006. Estimating Income Inequality in China Using Grouped Data and the Generalized Beta Distribution. UNU-WIDER Research Paper No. 2006/134. http://www.wider.unu.edu/publications/working-papers/researchpapers/en_GB/research-papers/ (accessed September 25, 2007) 
Energy Information Agency. 2004. File 11: Energy consumption. 2001 RECS Public Use Microdata Files.

http://www.eia.doe.gov/emeu/recs/recs2001/publicuse2001.html (accessed September 25, 2007)

Energy Information Agency. 2007a. Table S.4: Residential sector energy consumption estimates, 2004. http://www.eia.doe.gov/emeu/

states/sep_sum/plain_html/sum_btu_res.html (accessed September 25, 2007)

Energy Information Agency. 2007b. Total primary energy consumption (quadrillion btu), All countries 1980-2006. International Total Primary Energy Consumption and Energy Intensity. http://www.eia.doe. gov/emeu/international/energyconsumption.html (accessed April 7, 2009)

Gerber, L. 2007. A quintile rule for the Gini coefficient, Mathematics Magazine 80(2): 133-135.

Gini, C. 1921. Measurement of Inequality of Incomes. Economic Journal (31): 124-126.

Henri, Robert. 1960. The Art Spirit. Philadelphia and New York: J. B. Lippincott Company.

Hofheinz, P. 2001. Gates on technology, AIDS and why Malthus was wrong. ZDNet News, 29 January 2001. http://news.zdnet.com/21009595_22-527652.html (accessed October 3, 2007).

Jacobson, A., A. D. Milman, D. M. Kammen. 2007. Letting the (energy) Gini out of the bottle: Lorenz curves of cumulative electricity consumption and Gini coefficients as metrics of energy distribution and equity, Energy Policy (33) 1825-1832.

Kwok-chuen, K. 2007. Gini coefficient comparison misleading. On the Record, February 13. http://news.gov.hk/en/category/ontherecord/ 070212/html/070212en11001.htm (accessed September 25, 2007)

Leslie, R. A.. 1997. Exploring the Gini coefficient of Inequality with Derive. Electronic Proceedings of the 8th Annual International Conference on Technology in Collegiate Mathematics (1995). http://archives.math.utk.edu/ICTCM/EP-8.html (accessed September 25,2007 )

Lorenz, M. O. 1905. Methods of measuring the concentration of wealth. Publications of the American Statistical Association. Vol. 9 (New Series, No. 70) 209-219.

Palma, J. G. 2006. Globalizing Inequality: 'Centrifugal' and 'Centripetal' Forces at Work. DESA (Department of Economic and Social Affairs of the United Nations) Working Paper No. 35. 
http://www.un.org/esa/desa/papers/2006/wp35_2006.pdf (accessed September 25, 2007)

People's Daily Online. 2006. The limitation of the Gini coefficient in China. People's Daily Online, July 20th. http://english.peopledaily. com.cn/200607/20/ eng20060720_285083.html (accessed September 25, 2007)

Staples, M. 2005. Integrals and Equity. In Rethinking Mathematics: Teaching Social Justice by the Numbers, 103-106. Milwaukee, WI: Rethinking Schools, Ltd.

Teague, D. 2004. The Gini Index: Measuring Inequality. http://courses.ncssm.edu/math/Talks/PDFS/Gini (accessed September 25,2007 )

University of Texas Inequality Project. Measuring inequality (Powerpoint Presentation). http://utip.gov.utexas.edu/tutorials.html (accessed September 25, 2007)

Zorn, P. 2005. Measuring Inequality: The Gini Index. http://www.stolaf.edu/people/zorn/math128/gini.pdf (accessed September 25,2007$)$ 


\section{Appendix A: Energy Consumption by Country in 2004}

\begin{tabular}{|c|c||c|c|}
\hline $\begin{array}{c}\text { Percentile } \\
\text { of Country }\end{array}$ & $\begin{array}{c}\text { Percent } \\
\text { of Total }\end{array}$ & $\begin{array}{c}\text { Percentile } \\
\text { of Country }\end{array}$ & $\begin{array}{c}\text { Percent } \\
\text { of Total }\end{array}$ \\
\hline 0.005 & 0.00001 & 0.505 & 0.07316 \\
\hline 0.010 & 0.00004 & 0.510 & 0.07520 \\
\hline 0.014 & 0.00006 & 0.514 & 0.07725 \\
\hline 0.019 & 0.00010 & 0.519 & 0.07929 \\
\hline 0.024 & 0.00015 & 0.524 & 0.08136 \\
\hline 0.029 & 0.00019 & 0.529 & 0.08345 \\
\hline 0.033 & 0.00024 & 0.533 & 0.08555 \\
\hline 0.038 & 0.00030 & 0.538 & 0.08776 \\
\hline 0.043 & 0.00036 & 0.543 & 0.09001 \\
\hline 0.048 & 0.00042 & 0.548 & 0.09230 \\
\hline 0.052 & 0.00048 & 0.552 & 0.09466 \\
\hline 0.057 & 0.00054 & 0.557 & 0.09705 \\
\hline 0.062 & 0.00060 & 0.562 & 0.09952 \\
\hline 0.067 & 0.00068 & 0.567 & 0.10211 \\
\hline 0.071 & 0.00077 & 0.571 & 0.10469 \\
\hline 0.076 & 0.00085 & 0.576 & 0.10728 \\
\hline 0.081 & 0.00095 & 0.581 & 0.11005 \\
\hline 0.086 & 0.00104 & 0.586 & 0.11284 \\
\hline 0.090 & 0.00114 & 0.590 & 0.11576 \\
\hline 0.095 & 0.00124 & 0.595 & 0.11868 \\
\hline 0.100 & 0.00134 & 0.600 & 0.12165 \\
\hline 0.105 & 0.00145 & 0.605 & 0.12467 \\
\hline 0.110 & 0.00156 & 0.610 & 0.12768 \\
\hline 0.114 & 0.00167 & 0.614 & 0.13074 \\
\hline 0.119 & 0.00181 & 0.619 & 0.13381 \\
\hline 0.124 & 0.00196 & 0.624 & 0.13704 \\
\hline 0.129 & 0.00212 & 0.629 & 0.14046 \\
\hline 0.133 & 0.00228 & 0.633 & 0.14391 \\
\hline 0.138 & 0.00245 & 0.638 & 0.14744 \\
\hline 0.143 & 0.00263 & 0.643 & 0.15098 \\
\hline 0.148 & 0.00282 & 0.648 & 0.15455 \\
\hline 0.152 & 0.00302 & 0.652 & 0.15839 \\
\hline 0.157 & 0.00323 & 0.657 & 0.16229 \\
\hline 0.162 & 0.00344 & 0.662 & 0.16620 \\
\hline & & & \\
\hline
\end{tabular}




\begin{tabular}{|c|c|c|c|}
\hline $\begin{array}{l}\text { Percentile } \\
\text { of Country }\end{array}$ & $\begin{array}{l}\text { Percent } \\
\text { of Total }\end{array}$ & $\begin{array}{l}\text { Percentile } \\
\text { of Country }\end{array}$ & $\begin{array}{l}\text { Percent } \\
\text { of Total }\end{array}$ \\
\hline 0.167 & 0.00366 & 0.667 & 0.17023 \\
\hline 0.171 & 0.00388 & 0.671 & 0.17427 \\
\hline 0.176 & 0.00412 & 0.676 & 0.17855 \\
\hline 0.181 & 0.00438 & 0.681 & 0.18288 \\
\hline 0.186 & 0.00464 & 0.686 & 0.18723 \\
\hline 0.190 & 0.00491 & 0.690 & 0.19162 \\
\hline 0.195 & 0.00518 & 0.695 & 0.19623 \\
\hline 0.200 & 0.00548 & 0.700 & 0.20095 \\
\hline 0.205 & 0.00581 & 0.705 & 0.20567 \\
\hline 0.210 & 0.00614 & 0.710 & 0.21040 \\
\hline 0.214 & 0.00648 & 0.714 & 0.21546 \\
\hline 0.219 & 0.00682 & 0.719 & 0.22074 \\
\hline 0.224 & 0.00716 & 0.724 & 0.22602 \\
\hline 0.229 & 0.00753 & 0.729 & 0.23139 \\
\hline 0.233 & 0.00794 & 0.733 & 0.23684 \\
\hline 0.238 & 0.00839 & 0.738 & 0.24241 \\
\hline 0.243 & 0.00886 & 0.743 & 0.24803 \\
\hline 0.248 & 0.00936 & 0.748 & 0.25380 \\
\hline 0.252 & 0.00986 & 0.752 & 0.25957 \\
\hline 0.257 & 0.01037 & 0.757 & 0.26536 \\
\hline 0.262 & 0.01089 & 0.762 & 0.27119 \\
\hline 0.267 & 0.01146 & 0.767 & 0.27710 \\
\hline 0.271 & 0.01202 & 0.771 & 0.28312 \\
\hline 0.276 & 0.01259 & 0.776 & 0.28916 \\
\hline 0.281 & 0.01318 & 0.781 & 0.29522 \\
\hline 0.286 & 0.01377 & 0.786 & 0.30136 \\
\hline 0.290 & 0.01436 & 0.790 & 0.30768 \\
\hline 0.295 & 0.01498 & 0.795 & 0.31419 \\
\hline 0.300 & 0.01561 & 0.800 & 0.32072 \\
\hline 0.305 & 0.01630 & 0.805 & 0.32726 \\
\hline 0.310 & 0.01698 & 0.810 & 0.33385 \\
\hline 0.314 & 0.01767 & 0.814 & 0.34058 \\
\hline 0.319 & 0.01836 & 0.819 & 0.34738 \\
\hline 0.324 & 0.01911 & 0.824 & 0.35420 \\
\hline 0.329 & 0.01987 & 0.829 & 0.36103 \\
\hline 0.333 & 0.02068 & 0.833 & 0.36808 \\
\hline 0.338 & 0.02154 & 0.838 & 0.37513 \\
\hline 0.343 & 0.02247 & 0.843 & 0.38221 \\
\hline 0.348 & 0.02356 & 0.848 & 0.38950 \\
\hline 0.352 & 0.02469 & 0.852 & 0.39680 \\
\hline 0.357 & 0.02582 & 0.857 & 0.40411 \\
\hline
\end{tabular}




\begin{tabular}{|c|c||c|c|}
\hline $\begin{array}{c}\text { Percentile } \\
\text { of Country }\end{array}$ & $\begin{array}{c}\text { Percent } \\
\text { of Total }\end{array}$ & $\begin{array}{c}\text { Percentile } \\
\text { of Country }\end{array}$ & $\begin{array}{c}\text { Percent } \\
\text { of Total }\end{array}$ \\
\hline 0.362 & 0.02698 & 0.862 & 0.41172 \\
\hline 0.367 & 0.02815 & 0.867 & 0.41935 \\
\hline 0.371 & 0.02934 & 0.871 & 0.42719 \\
\hline 0.376 & 0.03054 & 0.876 & 0.43512 \\
\hline 0.381 & 0.03176 & 0.881 & 0.44341 \\
\hline 0.386 & 0.03301 & 0.886 & 0.45188 \\
\hline 0.390 & 0.03428 & 0.890 & 0.46044 \\
\hline 0.395 & 0.03558 & 0.895 & 0.46952 \\
\hline 0.400 & 0.03690 & 0.900 & 0.47876 \\
\hline 0.405 & 0.03823 & 0.905 & 0.48845 \\
\hline 0.410 & 0.03959 & 0.910 & 0.49876 \\
\hline 0.414 & 0.04096 & 0.914 & 0.50933 \\
\hline 0.419 & 0.04233 & 0.919 & 0.51992 \\
\hline 0.424 & 0.04371 & 0.924 & 0.53076 \\
\hline 0.429 & 0.04510 & 0.929 & 0.54179 \\
\hline 0.433 & 0.04651 & 0.933 & 0.55303 \\
\hline 0.438 & 0.04809 & 0.938 & 0.56708 \\
\hline 0.443 & 0.04968 & 0.943 & 0.58424 \\
\hline 0.448 & 0.05129 & 0.948 & 0.60163 \\
\hline 0.452 & 0.05292 & 0.952 & 0.61936 \\
\hline 0.457 & 0.05459 & 0.957 & 0.63759 \\
\hline 0.462 & 0.05629 & 0.962 & 0.65686 \\
\hline 0.467 & 0.05803 & 0.967 & 0.67747 \\
\hline 0.471 & 0.05983 & 0.971 & 0.69989 \\
\hline 0.476 & 0.06167 & 0.976 & 0.72496 \\
\hline 0.481 & 0.06351 & 0.981 & 0.75379 \\
\hline 0.486 & 0.06539 & 0.986 & 0.78825 \\
\hline 0.490 & 0.06727 & 0.990 & 0.82619 \\
\hline 0.495 & 0.06919 & 0.995 & 0.90590 \\
\hline 0.500 & 0.07114 & 1.000 & 0.99795 \\
\hline
\end{tabular}




\section{Appendix B: Energy Consumption by U.S. States in 2004}

\begin{tabular}{|c|c||c|c|}
\hline $\begin{array}{c}\text { Cum } \\
\text { \% State Rank }\end{array}$ & $\begin{array}{c}\text { Cum } \\
\text { \% Consumption }\end{array}$ & $\begin{array}{c}\text { Cum } \\
\text { \% State Rank }\end{array}$ & $\begin{array}{c}\text { Cum } \\
\text { \% Consumption }\end{array}$ \\
\hline 0.02 & 0.01 & 0.53 & 0.41 \\
\hline 0.04 & 0.02 & 0.55 & 0.43 \\
\hline 0.06 & 0.03 & 0.57 & 0.46 \\
\hline 0.08 & 0.04 & 0.59 & 0.48 \\
\hline 0.10 & 0.06 & 0.61 & 0.50 \\
\hline 0.12 & 0.07 & 0.63 & 0.52 \\
\hline 0.14 & 0.08 & 0.65 & 0.54 \\
\hline 0.16 & 0.10 & 0.67 & 0.56 \\
\hline 0.18 & 0.11 & 0.69 & 0.59 \\
\hline 0.20 & 0.12 & 0.71 & 0.61 \\
\hline 0.22 & 0.14 & 0.73 & 0.63 \\
\hline 0.24 & 0.15 & 0.75 & 0.66 \\
\hline 0.25 & 0.17 & 0.76 & 0.68 \\
\hline 0.27 & 0.18 & 0.78 & 0.71 \\
\hline 0.29 & 0.20 & 0.80 & 0.73 \\
\hline 0.31 & 0.21 & 0.82 & 0.76 \\
\hline 0.33 & 0.23 & 0.84 & 0.78 \\
\hline 0.35 & 0.24 & 0.86 & 0.81 \\
\hline 0.37 & 0.26 & 0.88 & 0.83 \\
\hline 0.39 & 0.28 & 0.90 & 0.86 \\
\hline 0.41 & 0.30 & 0.92 & 0.89 \\
\hline 0.43 & 0.31 & 0.94 & 0.91 \\
\hline 0.45 & 0.33 & 0.96 & 0.94 \\
\hline 0.47 & 0.35 & 0.98 & 0.97 \\
\hline 0.49 & 0.37 & 1.00 & 1.00 \\
\hline 0.51 & 0.39 & & \\
\hline & & & \\
\hline
\end{tabular}

